OPEN ACCESS

Edited by:

Julie A. Chowen,

Hospital Infantil Universitario Niño

Jesús, Spain

Reviewed by:

Jose Donato Jr.,

University of São Paulo, Brazil

Balazs Gaszner,

University of Pécs, Hungary

*Correspondence:

Sarah J. Spencer

sarah.spencer@rmit.edu.au

Specialty section:

This article was submitted to Neuroendocrine Science,

a section of the journal

Frontiers in Endocrinology

Received: 14 August 2017 Accepted: 11 October 2017 Published: 25 October 2017

Citation:

Ziko I, Sominsky L, Nguyen T-X, Yam K-Y, De Luca S, Korosi A and Spencer SJ (2017) Hyperleptinemia

in Neonatally Overfed Female

Rats Does Not Dysregulate

Feeding Circuitry.

Front. Endocrinol. 8:287.

doi: 10.3389/fendo.2017.00287

\section{Hyperleptinemia in Neonatally Overfed Female Rats Does Not Dysregulate Feeding Circuitry}

\author{
Ilvana Ziko', Luba Sominsky', Thai-Xinh Nguyen", Kit-Yi Yam², Simone De Luca1, \\ Aniko Korosi ${ }^{2}$ and Sarah J. Spencer ${ }^{1 *}$
}

'School of Health and Biomedical Sciences RMIT University, Melbourne, VIC, Australia, ${ }^{2}$ Swammerdam Institute for Life Sciences, Center for Neuroscience, University of Amsterdam, Amsterdam, Netherlands

Neonatal overfeeding during the first weeks of life in male rats is associated with a disruption in the peripheral and central leptin systems. Neonatally overfed male rats have increased circulating leptin in the first 2 weeks of life, which corresponds to an increase in body weight compared to normally fed counterparts. These effects are associated with a short-term disruption in the connectivity of neuropeptide Y (NPY), agouti-related peptide (AgRP), and pro-opiomelanocortin (POMC) neurons within the regions of the hypothalamus responsible for control of energy balance and food intake. Female rats that are overfed during the first weeks of their life experience similar changes in circulating leptin levels as well as in their body weight. However, it has not yet been studied whether these metabolic changes are associated with the same central effects as observed in males. Here, we hypothesized that hyperleptinemia associated with neonatal overfeeding would lead to changes in central feeding circuitry in females as it does in males. We assessed hypothalamic NPY, AgRP, and POMC gene expression and immunoreactivity at 7, 12, or 14 days of age, as well as neuronal activation in response to exogenous leptin in neonatally overfed and control female rats. Neonatally overfed female rats were hyperleptinemic and were heavier than controls. However, these metabolic changes were not mirrored centrally by changes in hypothalamic NPY, AGRP, and POMC fiber density. These findings are suggestive of sex differences in the effects of neonatal overfeeding and of differences in the ability of the female and male central systems to respond to changes in the early life nutritional environment.

Keywords: hypothalamus, nutrition, leptin, sex, satiety, obesity, neonatal

\section{INTRODUCTION}

Early life obesity is associated with increased risk of developing diabetes, cardiovascular complications and, consequently, increased rates of premature death $(1,2)$. Clinical studies have shown an association between the nutritional environment during early life and obesity, and obesity-related comorbidities, in adulthood (3). Similarly, in a neonatally overfed animal model representative of childhood obesity, both male and female rat pups raised in small litters, with greater access to their mothers' milk, experience significantly increased body weight, accompanied by obesity-related comorbidities in adulthood compared to pups raised in normal litters. These comorbidities include hypothalamic-pituitary-adrenal (HPA) axis dysfunction, impaired reproductive function, memory 
deficits, and many other effects (4-6). These neonatally overfed rats are also hyperleptinemic throughout life (7-12) and males, at least, are leptin resistant in the neonatal period (13-16).

In healthy adults, leptin, one of the main central regulators of energy balance and satiety, acts by activating hypothalamic pro-opiomelanocortin (POMC) and cocaine and amphetamineregulated transcript anorexigenic neurons and inhibiting neuropeptide Y (NPY) and agouti-related peptide (AgRP) orexigenic neurons to suppress feeding $(17,18)$. Recent data have shown, at least in males, an additional important role of leptin in the development of hypothalamic pathways that are related to feeding $(19,20)$. In rodents, a key developmental window for hypothalamic feeding circuitry occurs around postnatal day $(\mathrm{P})$ 4-16. This period coincides with naturally occurring high levels of leptin $(20,21)$, and this elevated leptin stimulates the growth of connections between the arcuate nucleus of the hypothalamus (ARC), paraventricular nucleus of the hypothalamus (PVN), dorsomedial hypothalamus, and lateral hypothalamus, a process that may later be curtailed by ghrelin $(20,22,23)$. In neonates, in the absence of leptin or ghrelin, or presence of a premature leptin surge or delayed ghrelin rise, neuronal connections between the relevant hypothalamic brain regions are impaired, leading to metabolic complications and obesity long term $(20,23,24)$.

Elevated circulating leptin from as early as P7, indicative of a premature leptin surge, can be seen with neonatal overfeeding $(12,14)$. As such, early life overnutrition in male mice leads to hyperleptinemia, and this is associated with early leptin resistance, accelerated weight gain, and increased fat mass in comparison to controls (25). These mice also have disturbances in the ghrelin system, including reduced central responses to exogenous ghrelin (26). Similarly, neonatal overfeeding in male rats leads to hyperleptinemia and suppressed circulating ghrelin, and this is associated with a dysregulation in NPY and AgRP neuronal networks within the ARC and PVN $(12,13,27)$. As shown in our previous work, neonatal overfeeding strongly increases the density of NPY and AgRP neuronal fibers in the ARC, while reducing the density of such fibers in the PVN, compared to normally fed controls (13). This profile is associated with reduced hypothalamic sensitivity to exogenous leptin and increased sensitivity to ghrelin, at least in the neonatal phase $(13,14,27)$. However, at least some of these effects may be specific to males (4).

Neonatal overfeeding leads to weight gain, exacerbated proinflammatory responses to immune challenge, and microglial priming in female rodents that are very similar to the effects seen in males (28-30). However, there are some noteworthy differences between the sexes. We have previously seen that neonatal overfeeding in females is associated with unique changes in the female HPA axis such that females, but not males, have exacerbated PVN responses to psychological stress accompanied by increased open arm exploration in the elevated plus maze, potentially related to an impaired ability of the female pituitary gland to respond to ghrelin $(4,10)$. Males, but not females, that are neonatally overfed also have disruptions in both circulating ghrelin and the ability of ghrelin to act at the hypothalamus to regulate feeding circuitry $(4,27)$; an effect that is present during the neonatal period but largely normalized by adulthood. Like males, neonatally overfed females are hyperleptinemic in juvenile and adult life $(5,12)$. However, it is currently unknown whether this hyperleptinemia in females leads to disruptions in hypothalamic feeding circuitry as it does in males, and the precedent for major sex differences in hypothalamic outcomes after neonatal overfeeding suggests a similarity to male responses cannot be assumed for females. We, therefore, aimed to determine if neonatal overfeeding-induced hyperleptinemia would compromise hypothalamic connectivity and hypothalamic responsiveness to leptin in females as it does in males.

\section{MATERIALS AND METHODS}

\section{Animals}

In these experiments, we used time-mated pregnant Wistar rats, obtained from the Animal Resources Centre, WA, Australia. On arrival at the RMIT University Animal Facility at day 14-16 of gestation, they were housed at $22^{\circ} \mathrm{C}, 12 \mathrm{~h}$ light/dark cycle $(0700$ hours to 1900 hours). We provided them with ad libitum pelleted standard rat chow and water. All procedures described here were conducted in accordance with the recommendations of the National Health and Medical Research Council Australia Code of Practice for the Care of Experimental Animals and the protocols were approved by the RMIT University Animal Ethics Committee.

\section{Litter Size Manipulation}

As we have previously described (10-12) on P0 (the day of birth), we removed all the rat pups from their dams and randomly redistributed them to new dams in litters of 12 (control litter; CL) or 4 (small litter; SL, neonatal overfeeding). Care was taken that none of the dams received any of their own pups. Each new litter was made up of equal numbers of males and females. Excess pups were culled by rapid decapitation. As we have previously seen, SL pups were significantly heavier than CL by P7 (10-12). Experimental animals were culled at P7, P12, or P14. Only females were used in the experiments described here. Data from the males of the same litters were used in other publications $(13,27)$. We derived all experimental groups from three or more litters, using a maximum of two pups from the same litter for an experimental treatment to control for maternal effects (31).

\section{Effects of Neonatal Overfeeding on Neonatal Circulating Leptin and Triglycerides}

On P7 or P14, we rapidly decapitated the animals and collected trunk blood for later assessment of plasma leptin and triglycerides. Whole blood was collected in EDTA-coated tubes, kept on ice and quickly centrifuged to separate the plasma. The plasma samples were aliquoted and stored at $-20^{\circ} \mathrm{C}$ avoiding freeze-thaw cycles until use.

To determine leptin concentrations in our samples, we performed a standard commercial leptin ELISA, following the manufacturer's instructions (Millipore, Ballerica, MA, USA). Intra-assay variability was $1.9-2.5 \% \mathrm{CV}$, inter-assay variability, $3.0-3.9 \% \mathrm{CV}$, and lower limit of detection, $0.04 \mathrm{ng} / \mathrm{ml}$. All compared samples were assayed in duplicates and processed in the same assay. 
To determine triglyceride concentrations in our samples, we performed a triglyceride assay (Cayman, Ann Arbor, MI, USA) according to the manufacturer's instructions. Intra-assay coefficient of variation was $1.34 \%$, inter-assay coefficient of variation was $3.17 \%$, and the lower limit of detection for this assay was $0.5 \mathrm{mg} / \mathrm{dl}$. All samples were assessed in duplicates and under the same conditions.

\section{Effects of Neonatal Overfeeding on Hypothalamic Gene Expression}

We used quantitative real time RT-PCR (qRT-PCR) to assess whether neonatal overfeeding alters hypothalamic gene expression of the Npy, Agrp, Pomc, or leptin receptor (Lepr) in the ARC and hypothalamus from the animals described above (see Table 1 for primer details). We dissected the brains into the right ARC and left hypothalamus not containing ARC as previously described (13). These samples were immediately snap-frozen in liquid nitrogen and stored at $-80^{\circ} \mathrm{C}$ until use. RNA was purified using QIAzol reagents and RNeasy Mini Kits (QIAGEN, Valencia, CA, USA). The RNA concentration was determined using a spectrophotometer (NanoDrop One, Thermo Scientific, Waltham, MA, USA). $1 \mu \mathrm{g}$ RNA was transcribed to cDNA using iScript cDNA synthesis kits (QIAGEN) according to manufacturer's instructions. A qRT-PCR Taqman Gene Expression Assay (Applied Biosystems, Mulgrave, VIC, Australia) was performed on an Applied Biosystems $^{\text {TM }}$ QuantStudio $^{\text {TM }} 7$ Flex qPCR System instrument (Life Technologies, Carlsbad, CA, USA), and the relative quantitative measure of the target gene expression was compared with an endogenous control, $\beta$-actin. $\beta$-actin was not significantly different between any of the groups. RNA expression was determined using the double delta $(C(t))$ equation $2^{-\Delta \Delta C(t)}$, where threshold cycle $[C(t)]$ values were the values at which fluorescence was first detected significantly above background, as previously described (13). Minus reverse-transcriptase (-RT), with omitted reverse transcriptase reactions, and no template controls (NTC), with omitted primer reactions, were run simultaneously with the samples in order to verify no genomic contamination was present. The mean $C(t)$ value of our $-\mathrm{RT}$ test samples for $\beta$-actin was more than 10 cycles different from the mean $C(t)$ value of our test samples, indicative of a twofold difference in the initial template amount, therefore, allowing us to assume $100 \%$ efficiency and the presence of negligible genomic DNA. NTC were undetermined $[>40 \mathrm{C}(t)]$, suggesting there was no DNA contamination. Data are presented as fold increase relative to P7 CLs. Since POMC cell bodies are found exclusively in two central nervous system nuclei, the ARC and the nucleus tractus solitarius $(32,33)$, we have not

TABLE 1 | Primer details for quantitative real-time PCR.

\begin{tabular}{lllc}
\hline Primer name & $\begin{array}{l}\text { NCBI reference } \\
\text { sequence }\end{array}$ & TaqMan assay ID & Product size \\
\hline Actb & NM_031144.2 & 4352340E & 91 \\
Lepr & NM_012596 & Rn01433205_ml & 94 \\
Npy & NM_012614.2 & Rn00561681_m1 & 63 \\
Agrp & NM_033650.1 & Rn01431703_g1 & 67 \\
Pomc & NM_139326.2 & Rn00595020_m1 & 92
\end{tabular}

examined POMC gene expression in the hypothalamic tissue that did not contain the ARC. Since hypothalamic AgRP neurons are only found in the ARC, we also did not examine AgRP in the hypothalamus not containing ARC.

\section{Neuronal Activation in Response to Exogenous Leptin}

To assess if neonatal overfeeding influences the ability of the hypothalamus to respond to circulating leptin, we assessed neuronal activation in response to $3 \mathrm{mg} / \mathrm{kg}$ i.p. leptin, or equivalent volume of $0.9 \%$ sterile saline, at P12. Pups were weighed immediately before and $45 \mathrm{~min}$ after injection. For immunohistochemical analysis of phosphorylated signal transducer and activator of transcription 3 (pSTAT3) as a marker of leptin-induced neuronal activation, we deeply anesthetized the pups with Lethabarb (150 mg/kg pentobarbitone sodium, i.p.) $45 \mathrm{~min}$ after injection and perfused them transcardially with phosphate-buffered saline (PBS: $4^{\circ} \mathrm{C}, \mathrm{pH} 7.4$ ), followed by $4 \%$ paraformaldehyde in PBS. Brains were removed and post-fixed for $24 \mathrm{~h}$ in the same fixative before placing them in $20 \%$ sucrose in $\mathrm{PBS}\left(4^{\circ} \mathrm{C}\right)$. The forebrains were then cut into $40 \mu \mathrm{m}$ coronal sections using a cryostat. Sections were cut into a one in five series and stored in sodium azide in PBS at $4^{\circ} \mathrm{C}$ until use. All our experiments were conducted between 0900 hours and 1300 hours to limit any effects of circadian rhythms in the measured parameters.

\section{Immunohistochemistry}

Sections through the hypothalamus were immunolabelled for NPY, AgRP, POMC, and pSTAT3. We incubated one of the five series of sections from each animal in primary antibody (NPY: 1:1,000; rabbit; overnight; $4^{\circ} \mathrm{C}$; Sigma-Aldrich, St. Louis, MO, USA. AgRP: 1:500; goat; $42 \mathrm{~h}$; room temperature; Neuromics Inc., MN, USA. POMC: 1:5,000; rabbit; overnight; $4^{\circ} \mathrm{C}$; Phoenix Pharmaceuticals, Burlingame, CA, USA. pSTAT3: 1:5,000; rabbit; overnight; $4^{\circ} \mathrm{C}$; Abcam, Cambridge, UK), followed by secondary antibody (NPY: 2 h; 1:500; Alexa-fluor 488 goat anti-rabbit; Thermo Scientific, Rockford, IL, USA. AgRP: 12 h 1:200 Alexafluor 594 rabbit anti-goat; Thermo Scientific. POMC: 1 h; 1:500; Alexa-fluor 488 goat anti-rabbit; Thermo Scientific. pSTAT3: $1.5 \mathrm{~h} ; 1: 500$; biotinylated anti-rabbit; Vector Laboratories, Burlingame, CA, USA). pSTAT3 sections were then incubated with avidin-biotin horseradish peroxidase (HRP) complex (ABC; 45 min; Vector Elite kit; Vector), followed by diaminobenzidine intensified with nickel to visualize the HRP activity. We stopped the reaction when the optimal contrast between the specific cellular and non-specific background labeling was observed. We air-dried the brain sections, dehydrated them in a series of alcohols, cleared them in histolene, and coverslipped. NPY, AgRP, and POMC sections were counterstained with DAPI for $15 \mathrm{~min}$ and mounted on slides with DAKO anti-fading solution.

The specificities of the antibodies used in our study have been previously validated by manufacturers and other researchers in pre-absorption and Western blotting experiments and further supported in our own validation experiments by incubation of experimental tissue without a primary or without a secondary antibody. Negligible positive labeling was seen in these negative 
controls (not shown). Positive labeling was confirmed in the brain regions of interest. More specifically, the NPY antibody (N9528) has been validated by the manufacturer and used in Ref. (34-37). The AgRP antibody (GT15023) has been validated by the manufacturer and used by Ref. $(35,38-43)$. The POMC antibody (H-029-30) specificity has been confirmed by Ref. (44) and used by Ref. (45-52). The pSTAT3 antibody (ab76315) has been previously validated by the manufacturer by Western blotting in HeLa cell lysate and used in the following publications (53-55).

Hypothalamic sections were assessed by an experimenter blinded to treatment groups. To visualize NPY, AgRP, and POMC labeling, photomicrographs were taken on an upright confocal laser-scanning microscope (Nikon Eclipse 90i, Tokyo, Japan) and visualized under 525/50 (NPY and POMC), 695/50 (AgRP), and 450/50 (DAPI) detection filters. ARC and PVN images were viewed under the $20 \times$ objective lens. NIS Elements Advanced Research Software (Nikon) was used to analyze fluorescence signal intensity within the ARC and PVN. Laser and detector levels were kept constant throughout the imaging. NPY, AgRP, and POMC cell fiber density were detected by the thresholding method as previously described (13). Four brain sections $120 \mu \mathrm{m}$ apart between 2.76 and $3.48 \mathrm{~mm}$ caudal to bregma per animal were analyzed. POMC-positive cells in the ARC were manually counted using Image J (National Institutes of Health, Bethesda, MD, USA). For the numbers of cells positive for pSTAT3, total immunoreactive cell numbers in the ARC and ventromedial hypothalamus (VMH) were counted. Summed counts of the four sections were taken as our sampled result.

\section{Data Analysis}

All data were analyzed using multifactorial analyses of variance (ANOVAs) with neonatal nutritional environment (CL/SL) and age (P7/14) or leptin treatment (saline/leptin) as between factors. Data were tested for homogeneity of variance and normality, using the Levene's test for Equality of Variance and the Shapiro-Wilks test, respectively, complemented by the assessment of skewness and kurtosis. Where significant interactions were found, we then performed Tukey post hoc tests. Immunoreactivity results were analyzed with Student's unpaired $t$-tests. Data are presented as the mean \pm SEM. Statistical significance was assumed when $p \leq 0.05$.

\section{RESULTS}

\section{Neonatal Overfeeding Leads to Accelerated Weight Gain}

In this cohort of rats, neonatal overfeeding led to early weight gain. Neonatally overfed (SL) rats were thus significantly heavier than controls [CL; significant age by litter size interaction: $F_{(1,32)}=30.37$, $p<0.001 ; n=8-10$; Figure 1A]. Post hoc analyses indicated that, by P14, the SL were significantly heavier than CL. These findings are similar to those we have previously published (10-12).

\section{Neonatal Overfeeding Effects on Circulating Leptin}

We have previously seen that circulating leptin levels are markedly higher in neonatally overfed than in control female (and
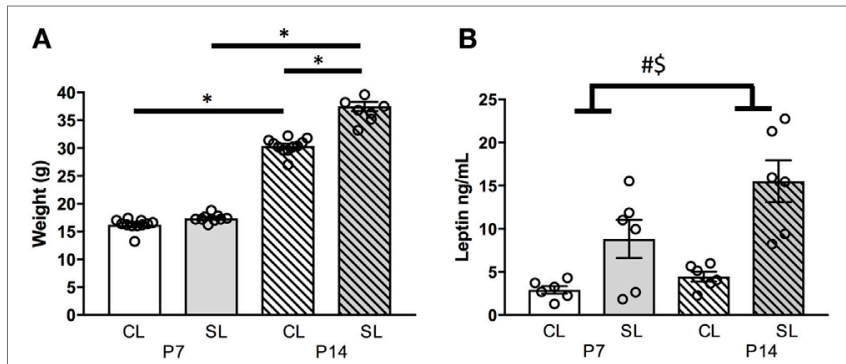

FIGURE 1 | Effects of neonatal overfeeding on the neonatal leptin system. (A) Body weights of control (CL) and small litter (SL) rats at postnatal day (P) 7 and 14, (B) plasma leptin concentrations at P7 and P14. Data are mean \pm SEM. $n=6-10$ per group. *Tukey's post hoc. \# significant main effect of litter size. $\$$ significant main effect of age. $p<0.05$. Note, in (A), error bars are overlapped by the data point circles.

male) rats during the neonatal period, and into adulthood, and we replicated the neonatal findings here $(5,12,13)$ [significant effect of litter size: $F_{(1,20)}=25.35, p<0.001$; significant effect of age: $F_{(1,20)}=6.01, p=0.024, n=6$ per group, Figure 1B].

\section{Neonatal Overfeeding Effects on Neonatal Hypothalamic Feeding-Related Gene Expression}

Neonatal overfeeding did not affect hypothalamic satiety-related genes measured here. However, significant age-related changes in these genes were observed. Expression of Lepr mRNA in the ARC and the hypothalamus was not affected by neonatal overfeeding, but it was significantly increased at P14 compared to P7 in the ARC [significant effect of age: $F_{(1,21)}=23.07, p<0.001 ; n=5-6$; Figure 2A] and the hypothalamus [significant effect of age: $F_{(1,21)}=13.83, p<0.001 ; n=5-7$; Figure 2B] in both groups.

Npy mRNA expression in the ARC was not affected by neonatal overfeeding, but it was significantly increased at P14 compared to P7 [significant effect of age: $F_{(1,19)}=5.84, p=0.026 ; n=5-6$; Figure 2C]. In the hypothalamus, neonatal overfeeding had no effect on Npy expression (Figure 2D). Agrp mRNA expression in the ARC was not affected by neonatal overfeeding; however, it was significantly increased at P14 compared to P7 [significant effect of age: $F_{(1,19)}=12.86, p=0.002 ; n=5-6$; Figure $\left.2 E\right]$. Neonatal overfeeding also did not affect Pomc mRNA expression in the ARC. However, again, there was a significant increase of Pomc mRNA in P14 ARC compared to P7 [significant effect of age: $F_{(1,17)}=6.16, p=0.024 ; n=5-6$; Figure 2F].

\section{Neonatal Overfeeding Effects on Neonatal Hypothalamic NPY, AgRP, POMC}

To determine if the elevated leptin in neonatally overfed females was associated with a disruption of hypothalamic NPY, AgRP, and POMC as previously described in males (13), we examined NPY fibers (ARC: Figures 3A,B, PVN: Figures 3C,D), AgRP fibers (ARC: Figures 3E,F, PVN: Figures 3G,H), and POMC-positive cells in the ARC (Figures 3I,J) and POMC fibers in the PVN 

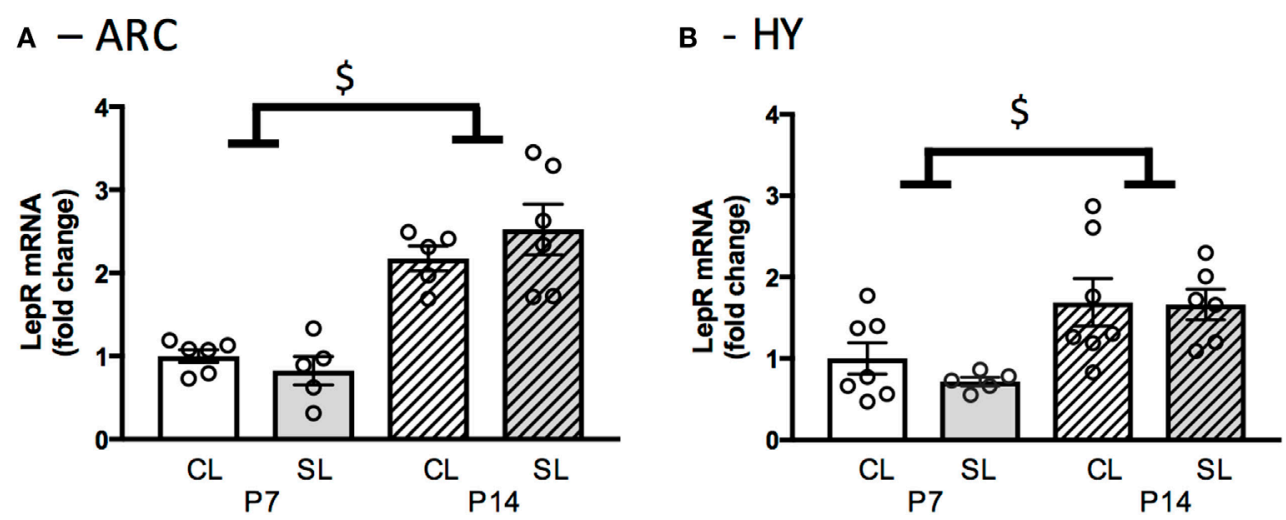

c - ARC

D $-\mathrm{HY}$
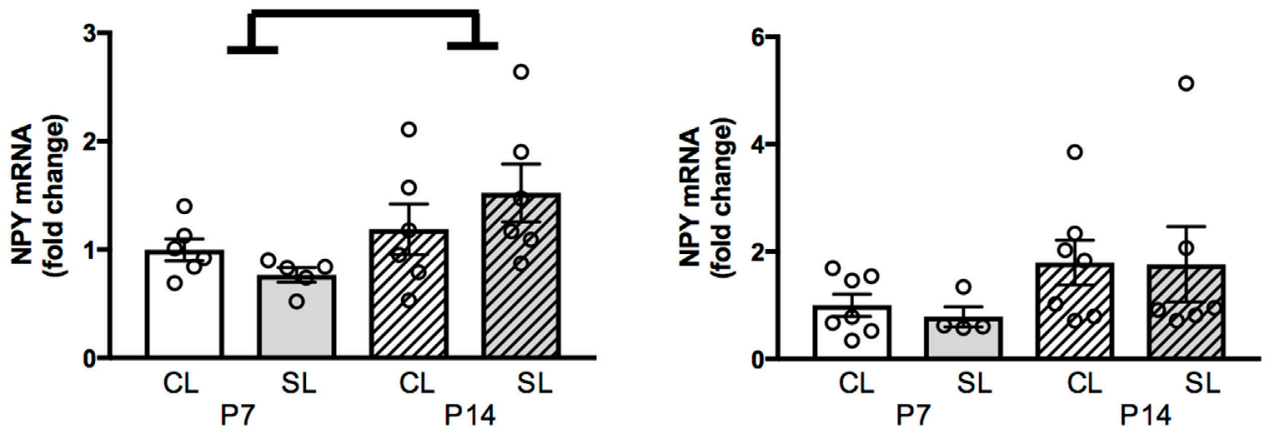

$E-A R C$

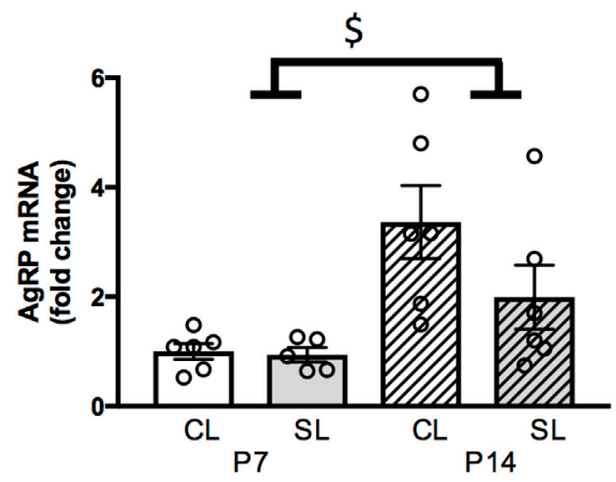

F - ARC

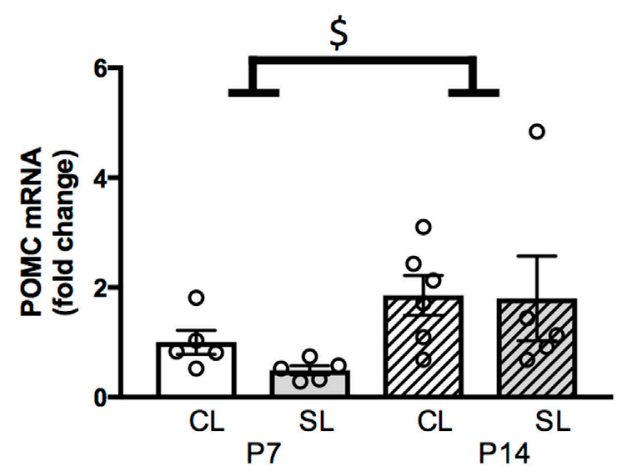

FIGURE 2 | Neonatal overfeeding effects on neonatal hypothalamic feeding-related gene expression. (A) Leptin receptor expression in the arcuate nucleus of the hypothalamus (ARC) and (B) hypothalamus (HY) of control (CL) and small litter (SL) rats at postnatal day (P) 7 and P14. (C) Neuropeptide Y (NPY) gene expression in the ARC and (D) in the HY. (E) Agouti-related peptide (AgRP) gene expression in the ARC. (F) Pro-opiomelanocortin (POMC) gene expression in the ARC. Data are mean \pm SEM. $n=5-6$ per group. ${ }^{\$}$ Significant main effect of age. $p<0.05$.

(Figures 3K,L) at P12. Neonatal overfeeding did not affect NPY, AgRP, and POMC immunoreactive fibers or POMC immunoreactive cells in these regions ( $n=5-6$ per group).

\section{Neonatal Overfeeding Effects on Neonatal Hypothalamic Responses to Leptin}

We next tested if neonatal overfeeding alters the ability of the neonatal hypothalamus to respond to a leptin signal by giving the pups a single injection of leptin, or saline, on P12 and measuring weight changes as well as hypothalamic pSTAT3 expression. There were no differences in the weight gain after $45 \mathrm{~min}$ in any of the groups (data not shown). Leptin stimulated an increase in the number of pStat 3 positive cells in the ARC, as expected $\left[F_{(1,17)}=101.30, p<0.001 ; n=5-6\right]$. There was also a significant main effect of litter size $\left[F_{(1,17)}=7.36, p=0.015\right.$; Figures $\left.4 A, C, D\right]$, but no interaction. In the $\mathrm{VMH}$, leptin injection also induced a significant increase in pSTAT3-positive cells [main effect of leptin: $F_{(1,17)}=42.84, p<0.001 ; n=5-6$; Figures 4B,C,E]. 


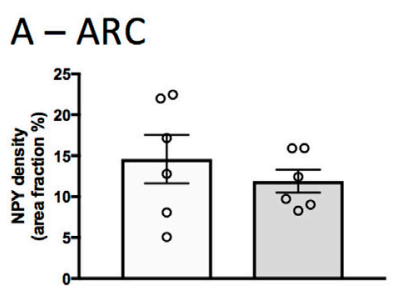

$\mathrm{CL}$

C - PVN

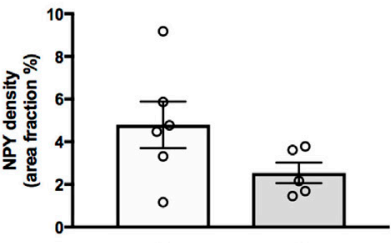

$\mathrm{CL}$

SL

$E-A R C$

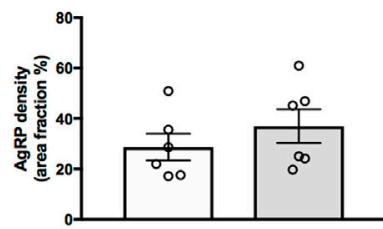

CL $\quad \mathrm{SL}$

G - PVN

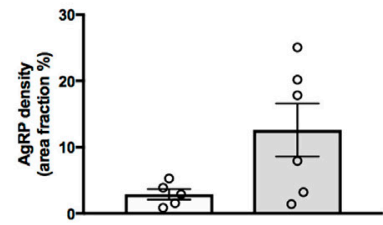

$\mathrm{CL}$

$\mathrm{SL}$

I-ARC

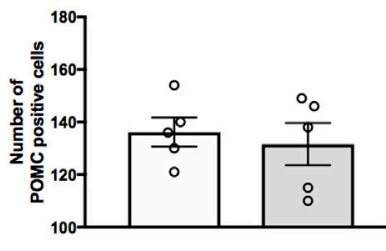

$\mathrm{CL}$

SL

$\mathrm{K}-\mathrm{PVN}$

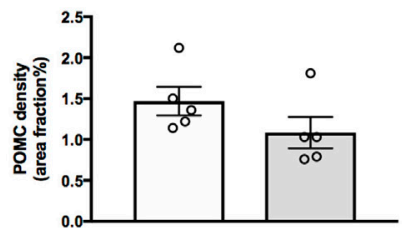

$\mathrm{CL}$
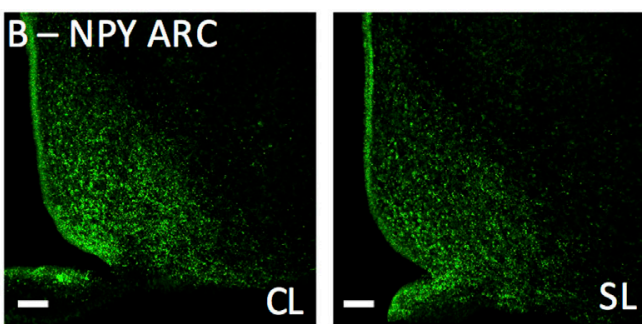

D-NPY PVN

CL
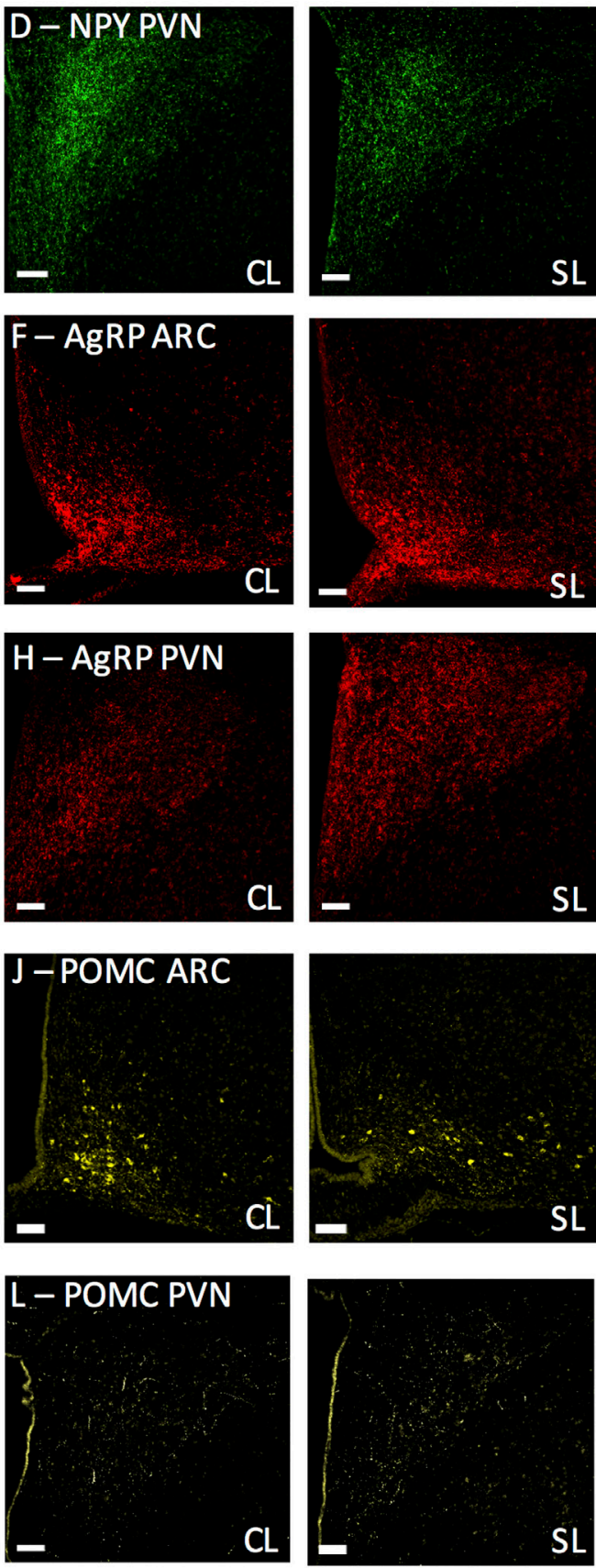

FIGURE 3 | Neonatal overfeeding effects on neonatal hypothalamic circuitry. (A,B) neuropeptide $\mathrm{Y}$ (NPY) labeling in the arcuate nucleus of the hypothalamus (ARC) and $(\mathbf{C}, \mathbf{D})$ paraventricular nucleus of the hypothalamus (PVN) of control (CL) and small litter (SL) rats at postnatal day (P) 12. (E,F) Agouti-related peptide (AgRP) labeling in the ARC and (G,H) PVN. (I,J) Proopiomelanocortin (POMC) positive cells in the ARC. (K,L) POMC labeling in the PVN. Representative photomicrographs showing NPY labeling in the (B) ARC and (D) PVN, AgRP labeling in the (F) ARC, and (H) PVN, POMC labeling in the (J) ARC and (L) PVN. Scale bars = $100 \mu \mathrm{m}$. Data are mean \pm SEM. $n=5-6$ per group. 

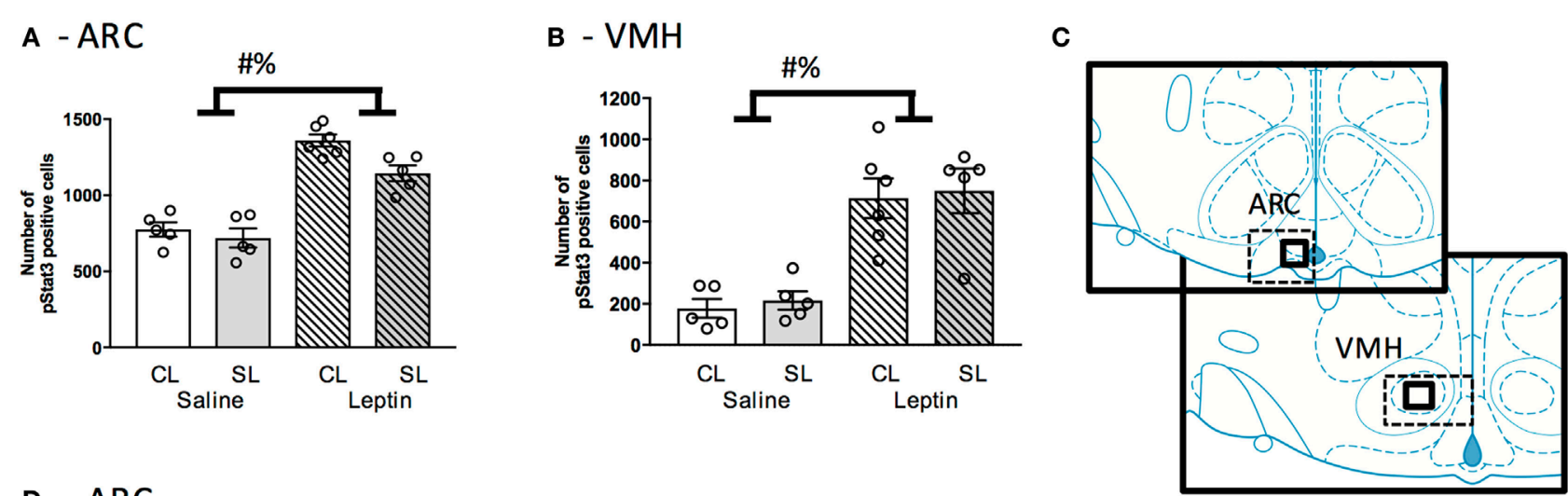

\section{D - ARC}
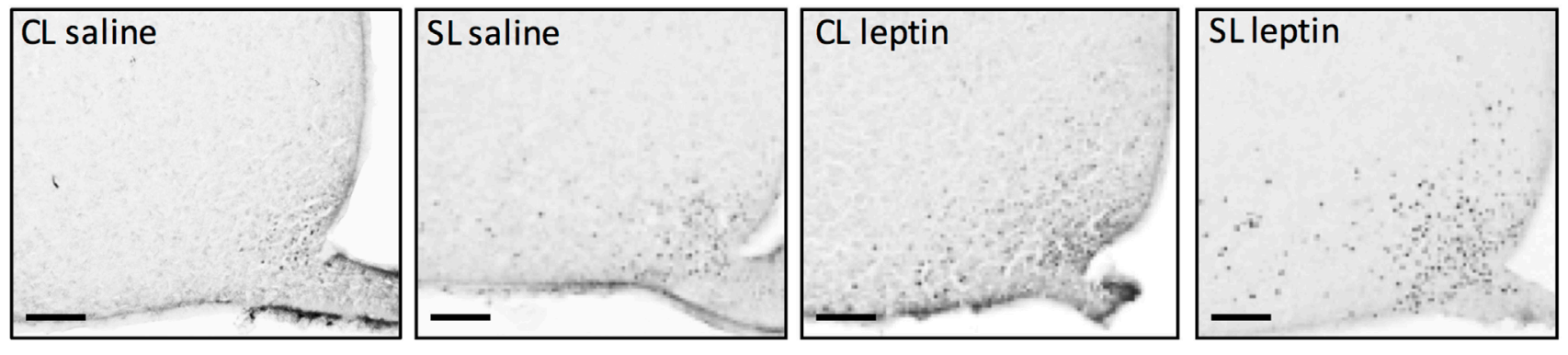

\section{E - VMH}
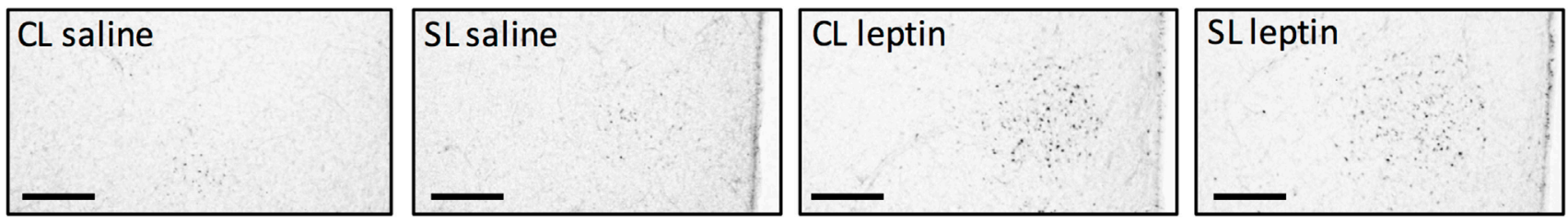

FIGURE 4 | Neonatal overfeeding effects on neonatal hypothalamic responses to leptin. (A) Arcuate nucleus of the hypothalamus (ARC) and (B) ventromedial hypothalamus $(\mathrm{VMH})$ neuronal activation in response to leptin injection at postnatal day (P) 12 in control (CL) and small litter (SL) rats as assessed by numbers of signal transducer and activator of transcription (pSTAT3) positive cells. The sum of cell counts in four sections was plotted. (C) Schematic diagram adapted from Paxinos and Watson illustrating the regions of interest. Thick-line boxes are representative of analyzed regions, dotted-line boxes are representative of the photomicrographs. Representative photomicrographs of pSTAT3 after a saline or leptin injection in the (D) ARC (scale bars = $100 \mu \mathrm{m})$ and (E) VMH (scale bars $=200 \mu \mathrm{m})$. Data are mean \pm SEM. $n=5-6$ per group. \# significant main effect of litter size. \% significant main effect of leptin. $p<0.05$.

\section{Neonatal Overfeeding Effects on Neonatal Circulating Triglycerides}

Since neonatal overfeeding had minimal effects on hypothalamic feeding circuitry in female rats despite inducing pronounced neonatal hyperleptinemia, we next examined circulating triglyceride levels. Triglycerides have been shown to mediate leptin transport to the brain (56). We, therefore, hypothesized neonatal overfeeding might lead to elevated circulating triglycerides that could reduce leptin transport to the brain and thus reduce the effects of hyperleptinemia. In this regard, neonatal overfeeding led to an increase in circulating triglyceride levels [significant effect of litter size: $F_{(1,20)}=5.43, p=0.030 ; n=6$ per group, Figure 5], without an effect of age.

\section{DISCUSSION}

The early life nutritional environment plays a crucial role in metabolism and neurodevelopment. Here, for the first time, we show that neonatal overfeeding in females, despite hyperleptinemia and a corresponding increased body weight, does not affect NPY, AgRP, and POMC mRNA or protein in the hypothalamic circuitry responsible for feeding and metabolic control. These observations are different from findings previously shown in neonatally overfed males, where overfeeding leading to hyperleptinemia and increased body weight are associated with short-term disruption of hypothalamic neuronal wiring responsible for metabolic regulation $(13,26)$.

Naturally occurring high circulating levels of leptin are seen at approximately $\mathrm{P} 4$ to $\mathrm{P} 16$ in mice (57) and $\mathrm{P} 4$ to $\mathrm{P} 14$ in rats (58) with a peak at P10 (59), they then normalize toward adult levels after weaning (21). Such an increase in leptin levels is not associated with acute changes in food intake, but is reflective of leptin's neurodevelopmental role in stimulating the growth of hypothalamic connections between the ARC and other hypothalamic regions that are ultimately responsible for controlling energy balance (21). Disruptions to this leptin surge can permanently impact upon the development of these hypothalamic connections and 


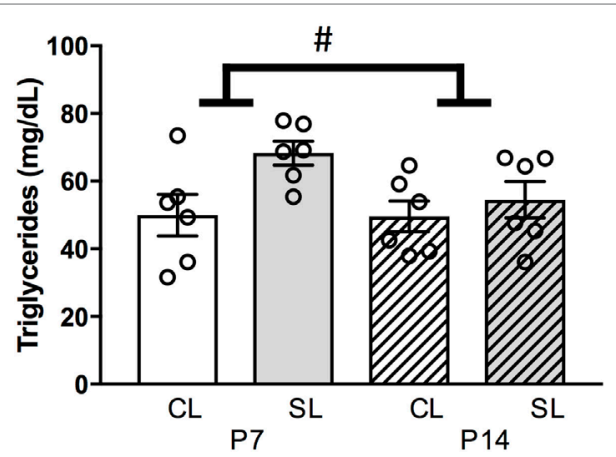

FIGURE 5 | Neonatal overfeeding effects on neonatal circulating triglycerides. Plasma triglyceride concentration at postnatal day (P)7 and P14 in control $(\mathrm{CL})$ and small litter $(\mathrm{SL})$ rats. Data are mean \pm SEM. $n=6$ per group. \# significant main effect of litter size. $p<0.05$.

lead to aberrant feeding behavior and metabolism throughout life $(20,22)$. For example, leptin-deficient (ob/ob) male mice have a lack of innervation developing from the ARC toward the PVN and other hypothalamic regions (20), deficits that can be repaired by restoration of normal neonatal leptin levels (20). Being suckled in a small litter during the first 3 weeks of life in mice and rats can similarly disrupt hypothalamic circuitry. Thus, early life overfeeding increases body weight, fat mass, and circulating leptin in comparison to the normally fed. In males, this hyperleptinemia is associated with a disruption in NPY and AgRP fibers and leads to an obese phenotype that is maintained throughout life $(12,14,60,61)$.

It is important to note that most of the current knowledge in regards to the effects of postnatal overfeeding on hypothalamic neuronal development derives from observations in male rodents. However, changes in the neonatal leptin availability as a result of overfeeding appear to induce sex-dependent effects on the development of hypothalamic neuronal connectivity. We observe here that in females, similarly to males, neonatal overfeeding is associated with exacerbated hyperleptinemia during the neonatal leptin surge period, and this is followed by an increase in body weight. However, neonatally overfed females are still responsive to leptin and, importantly, there is no effect of female neonatal overfeeding on the mRNA levels of the Lepr, or in the changes in the gene or protein expression of NPY, AgRP, or POMC in the hypothalamus. The ARC of small litter animals showed decreased sensitivity to leptin, although there was no interaction between litter size and leptin. However, the magnitude of this difference is small, of the order of $15 \%$ of the response in the case of the leptin treated. We also do not see this difference in the VMH. We observe here that neonatally overfed females have increased circulating triglyceride levels relative to controls. It is thus possible that the hyperleptinemia in females is compensated for by elevated blood triglycerides restricting leptin's access to the brain in the neonatally overfed (56), which may also account for the minor reduction in the number of pSTAT3-positive cells in the ARC of these animals. However, this observation does not explain why males are not also resistant to the effects of excess leptin, since we would expect males to also have increased circulating triglyceride levels when neonatally overfed. There is some evidence of sex differences in triglyceride levels in adults after an early food restriction. For instance, there are elevated triglycerides in perinatally foodrestricted females relative to males (62), while other perinatal insults, such as maternal deprivation (63), have been shown to increase circulating triglycerides in adult males, but decrease them in females. However, no direct sex comparison has yet been made during the neonatal phase.

We have previously seen sex differences in the way that neonatally overfed rats control energy expenditure. Female rats that are overfed as neonates remain fat into adulthood and they do this not by overeating, but by reducing energy expenditure, probably due to reduced activation of brown adipose tissue (BAT) during the first half of the dark phase (12). BAT is responsible for the conversion of energy from food into heat primarily via uncoupling protein (64) and neonatally overfed females are unable to optimally convert BAT into energy and show reduced energy consumption at the juvenile stage, with normalization of function in adulthood. This disruption of BAT function at the juvenile stage is not evident in the neonatally overfed males (12). BAT thermogenesis is reduced in neonatally overfed females until P30 but, by adulthood, BAT thermogenesis normalizes to controls levels (12), a possible explanation of this is how these females retain an elevated body weight without any changes in their hypothalamic feeding networks.

Another potential reason for these sex-dependent effects of neonatal overfeeding may lie in the organization of sexually dimorphic neural pathways. Sex steroid hormones such as estrogen and testosterone, in combination with neurotrophins, are able to regulate formation of sexually dimorphic circuits by affecting axonal guidance and synaptogenesis [reviewed in Ref. (65)]. Significant hormonal changes in the central nervous system in rodents happen partly due to the gonadal steroid hormones during sexual differentiation (66). For instance, sexually dimorphic nucleus, a cluster of cells in the preoptic area of the hypothalamus responsible for controlling sexual behavior by affecting sex hormones such as testosterone and estrogens in rats, develops from as early as P1. By P8, this region is almost twice the size in males as that of females [reviewed in Ref. (67)]. Conversely, the anteroventral periventricular nucleus, a region of the hypothalamus responsible for the pulsatile release of gonadotropin releasing hormone, covers a larger area and consists of a larger number of neurons in females than in males [reviewed in Ref. (65)]. Testosterone and estrogens influence energy homeostasis at least partially via hormonal receptors, which are colocalized with hunger or satiety neuropeptides located in the hypothalamus [reviewed in Ref. (68)], suggesting that sexual dimorphism affects the development of hypothalamic regions that control energy balance and may thus also be reflected in the differential effects of neonatal overfeeding on hypothalamic appetite-regulatory circuitry.

Overall, our results suggest females overfed during early life, despite being hyperleptinemic and experiencing an obesogenic phenotype, are not acutely affected in their central neuronal connectivity responsible for metabolic control. These results contrast with our and others' previous findings in males. These findings are potentially reflective of differences in how females and males adapt to early life environmental dietary challenges. Our work further 
highlights that it is important not to assume female physiology from male data and that different physiological mechanisms may lead to a similar phenotypic outcome, in this case, excess body weight.

\section{ETHICS STATEMENT}

All procedures described here were conducted in accordance with the recommendations of the National Health and Medical Research Council Australia Code of Practice for the Care of Experimental Animals and the protocols were approved by the RMIT University Animal Ethics Committee.

\section{AUTHOR CONTRIBUTIONS}

SS conceived of and designed the work. IZ, LS, T-XN, K-YY, SL, $\mathrm{AK}$, SS (i.e., all authors) made substantial contributions to the

\section{REFERENCES}

1. Franks PW, Hanson RL, Knowler WC, Sievers ML, Bennett PH, Looker HC. Childhood obesity, other cardiovascular risk factors, and premature death. N Engl J Med (2010) 362(6):485-93. doi:10.1056/NEJMoa0904130

2. Barton M. Childhood obesity: a life-long health risk. Acta Pharmacol Sin (2012) 33(2):189-93. doi:10.1038/aps.2011.204

3. Dunger DB, Salgin B, Ong KK. Session 7: early nutrition and later health early developmental pathways of obesity and diabetes risk. Proc Nutr Soc (2007) 66(3):451-7. doi:10.1017/S0029665107005721

4. Sominsky L, Ziko I, Spencer SJ. Neonatal overfeeding disrupts pituitary ghrelin signalling in female rats long-term; Implications for the stress response. PLoS One (2017) 12(3):e0173498. doi:10.1371/journal.pone.0173498

5. Sominsky L, Ziko I, Soch A, Smith JT, Spencer SJ. Neonatal overfeeding induces early decline of the ovarian reserve: implications for the role of leptin. Mol Cell Endocrinol (2016) 431:24-35. doi:10.1016/j.mce.2016.05.001

6. De Luca SN, Ziko I, Sominsky L, Nguyen JC, Dinan T, Miller AA, et al. Early life overfeeding impairs spatial memory performance by reducing microglial sensitivity to learning. J Neuroinflammation (2016) 13(1):112. doi:10.1186/ s12974-016-0578-7

7. Fiorotto ML, Burrin DG, Perez M, Reeds PJ. Intake and use of milk nutrients by rat pups suckled in small, medium, or large litters. Am J Physiol (1991) 260(6 Pt 2):R1104-13.

8. Plagemann A, Heidrich I, Gotz F, Rohde W, Dorner G. Obesity and enhanced diabetes and cardiovascular risk in adult rats due to early postnatal overfeeding. Exp Clin Endocrinol (1992) 99(3):154-8. doi:10.1055/s-0029-1211159

9. Morris MJ, Velkoska E, Cole TJ. Central and peripheral contributions to obesity-associated hypertension: impact of early overnourishment. Exp Physiol (2005) 90(5):697-702. doi:10.1113/expphysiol.2005.030783

10. Spencer SJ, Tilbrook A. Neonatal overfeeding alters adult anxiety and stress responsiveness. Psychoneuroendocrinology (2009) 34(8):1133-43. doi:10.1016/j.psyneuen.2009.02.013

11. Smith JT, Spencer SJ. Preweaning over- and underfeeding alters onset of puberty in the rat without affecting kisspeptin. Biol Reprod (2012) 86(5):145,1-8. doi:10.1095/biolreprod.111.097758

12. Stefanidis A, Spencer SJ. Effects of neonatal overfeeding on juvenile and adult feeding and energy expenditure in the rat. PLoS One (2012) 7(12):e52130. doi:10.1371/journal.pone.0052130

13. Sominsky L, Ziko I, Nguyen TX, Quach J, Spencer SJ. Hypothalamic effects of neonatal diet: reversible and only partially leptin dependent. J Endocrinol (2017) 234(1):41-56. doi:10.1530/JOE-16-0631

14. Lopez M, Tovar S, Vazquez MJ, Nogueiras R, Seoane LM, Garcia M, et al. Perinatal overfeeding in rats results in increased levels of plasma leptin but unchanged cerebrospinal leptin in adulthood. Int J Obesity (Lond) (2007) 31(2):371-7. doi:10.1038/sj.ijo.0803425

15. Wang H, Ji J, Yu Y, Wei X, Chai S, Liu D, et al. Neonatal overfeeding in female mice predisposes the development of obesity in their male offspring via altered acquisition, analysis, and/or interpretation of data for the work. IZ, LS, and SS drafted the work. All authors critically revised it for important intellectual content. All authors give final approval of the version to be published. All authors agree to be accountable for all aspects of the work in ensuring that questions related to the accuracy or integrity of any part of the work are appropriately investigated and resolved.

\section{FUNDING}

This work was supported by funding from by a National Health and Medical Research Council Career Development Fellowship, a Club Melbourne Fellowship and a Brain Foundation Research Gift to SS, and an NWO Meervoud and NWO Food Cognition and Behavior (NWO-FCB), JPI-Nutricog to AK.

central leptin signalling. J Neuroendocrinol (2015) 27(7):600-8. doi:10.1111/ jne. 12281

16. Schmidt I, Fritz A, Scholch C, Schneider D, Simon E, Plagemann A. The effect of leptin treatment on the development of obesity in overfed suckling Wistar rats. Int J Obes Relat Metab Disord (2001) 25(8):1168-74. doi:10.1038/ sj.ijo.0801669

17. Cowley MA, Smart JL, Rubinstein M, Cerdan MG, Diano S, Horvath TL, et al. Leptin activates anorexigenic POMC neurons through a neural network in the arcuate nucleus. Nature (2001) 411(6836):480-4. doi:10.1038/ 35078085

18. Pinto S, Roseberry AG, Liu H, Diano S, Shanabrough M, Cai X, et al. Rapid rewiring of arcuate nucleus feeding circuits by leptin. Science (2004) 304(5667):110-5. doi:10.1126/science.1089459

19. Bouret SG, Simerly RB. Development of leptin-sensitive circuits. J Neuroendocrinol(2007) 19(8):575-82. doi:10.1111/j.1365-2826.2007.01563.x

20. Bouret SG, Draper SJ, Simerly RB. Formation of projection pathways from the arcuate nucleus of the hypothalamus to hypothalamic regions implicated in the neural control of feeding behavior in mice. J Neurosci (2004) 24(11):2797-805. doi:10.1523/JNEUROSCI.5369-03.2004

21. Ahima RS, Prabakaran D, Flier JS. Postnatal leptin surge and regulation of circadian rhythm of leptin by feeding. Implications for energy homeostasis and neuroendocrine function. J Clin Invest (1998) 101(5):1020-7. doi:10.1172/ JCI1176

22. Bouret SG, Draper SJ, Simerly RB. Trophic action of leptin on hypothalamic neurons that regulate feeding. Science (2004) 304(5667):108-10. doi:10.1126/ science. 1095004

23. Steculorum SM, Collden G, Coupe B, Croizier S, Lockie S, Andrews ZB, et al. Neonatal ghrelin programs development of hypothalamic feeding circuits. J Clin Invest (2015) 125(2):846-58. doi:10.1172/JCI73688

24. Yura S, Itoh H, Sagawa N, Yamamoto H, Masuzaki H, Nakao K, et al. Role of premature leptin surge in obesity resulting from intrauterine undernutrition. Cell Metab (2005) 1(6):371-8. doi:10.1016/j.cmet.2005.05.005

25. Glavas MM, Kirigiti MA, Xiao XQ, Enriori PJ, Fisher SK, Evans AE, et al. Early overnutrition results in early-onset arcuate leptin resistance and increased sensitivity to high-fat diet. Endocrinology (2010) 151(4):1598-610. doi:10.1210/en.2009-1295

26. Collden G, Balland E, Parkash J, Caron E, Langlet F, Prevot V, et al. Neonatal overnutrition causes early alterations in the central response to peripheral ghrelin. Mol Metab (2015) 4(1):15-24. doi:10.1016/j.molmet.2014.10.003

27. Sominsky L, Ziko I, Nguyen TX, Andrews ZB, Spencer SJ. Early life disruption to the ghrelin system with over-eating is resolved in adulthood in male rats. Neuropharmacology (2016) 113(Pt A):21-30. doi:10.1016/j. neuropharm.2016.09.023

28. Cai G, Ziko I, Barwood J, Soch A, Sominsky L, Molero JC, et al. Overfeeding during a critical postnatal period exacerbates hypothalamic-pituitary-adrenal axis responses to immune challenge: a role for adrenal melanocortin 2 receptors. Sci Rep (2016) 6:21097. doi:10.1038/srep21097 
29. Clarke MA, Stefanidis A, Spencer SJ. Postnatal overfeeding leads to obesity and exacerbated febrile responses to lipopolysaccharide throughout life. J Neuroendocrinol (2012) 24(3):511-24. doi:10.1111/j.1365-2826.2011.02269.x

30. Ziko I, De Luca S, Dinan T, Barwood JM, Sominsky L, Cai G, et al. Neonatal overfeeding alters hypothalamic microglial profiles and central responses to immune challenge long-term. Brain Behav Immun (2014) 41:32-43. doi:10.1016/j.bbi.2014.06.014

31. Spencer SJ, Meyer U. Perinatal programming by inflammation. Brain Behav Immun (2017) 63:1-7. doi:10.1016/j.bbi.2017.02.007

32. Joseph SA, Pilcher WH, Bennett-Clarke C. Immunocytochemical localization of ACTH perikarya in nucleus tractus solitarius: evidence for a second opiocortin neuronal system. Neurosci Lett (1983) 38(3):221-5. doi:10.1016/0304-3940(83)90372-5

33. Cone RD. Anatomy and regulation of the central melanocortin system. Nat Neurosci (2005) 8(5):571-8. doi:10.1038/nn1455

34. Garcia AP, Palou M, Sanchez J, Priego T, Palou A, Pico C. Moderate caloric restriction during gestation in rats alters adipose tissue sympathetic innervation and later adiposity in offspring. PLoS One (2011) 6(2):e17313. doi:10.1371/journal.pone.0017313

35. Kobelt P, Wisser AS, Stengel A, Goebel M, Inhoff T, Noetzel S, et al. Peripheral injection of ghrelin induces Fos expression in the dorsomedial hypothalamic nucleus in rats. Brain Res (2008) 1204:77-86. doi:10.1016/j. brainres.2008.01.054

36. Konieczna J, Garcia AP, Sanchez J, Palou M, Palou A, Pico C. Oral leptin treatment in suckling rats ameliorates detrimental effects in hypothalamic structure and function caused by maternal caloric restriction during gestation. PLoS One (2013) 8(11):e81906. doi:10.1371/journal.pone.0081906

37. Pekala D, Blasiak T, Raastad M, Lewandowski MH. The influence of orexins on the firing rate and pattern of rat intergeniculate leaflet neurons - electrophysiological and immunohistological studies. Eur J Neurosci (2011) 34(9):1406-18. doi:10.1111/j.1460-9568.2011.07868.x

38. Betley JN, Xu S, Cao ZFH, Gong R, Magnus CJ, Yu Y, et al. Neurons for hunger and thirst transmit a negative-valence teaching signal. Nature (2015) 521(7551):180-5. doi:10.1038/nature14416

39. Cao Y, Nakata M, Okamoto S, Takano E, Yada T, Minokoshi Y, et al. PDK1Foxo1 in agouti-related peptide neurons regulates energy homeostasis by modulating food intake and energy expenditure. PLoS One (2011) 6(4):e18324. doi:10.1371/journal.pone.0018324

40. Garfield AS, Li C, Madara JC, Shah BP, Webber E, Steger JS, et al. A neural basis for melanocortin-4 receptor-regulated appetite. Nat Neurosci (2015) 18(6):863-71. doi:10.1038/nn.4011

41. Kim ER, Lew PS, Spirkina A, Mizuno TM. Xenin-induced feeding suppression is not mediated through the activation of central extracellular signal-regulated kinase signaling in mice. Behav Brain Res (2016) 312:118-26. doi:10.1016/j. bbr.2016.06.026

42. Li AJ, Wiater MF, Oostrom MT, Smith BR, Wang Q, Dinh TT, et al. Leptinsensitive neurons in the arcuate nuclei contribute to endogenous feeding rhythms. Am J Physiol Regul Integr Comp Physiol (2012) 302(11):R1313-26. doi:10.1152/ajpregu.00086.2012

43. Shibata M, Banno R, Sugiyama M, Tominaga T, Onoue T, Tsunekawa $T$, et al. AgRP neuron-specific deletion of glucocorticoid receptor leads to increased energy expenditure and decreased body weight in female mice on a high-fat diet. Endocrinology (2016) 157(4):1457-66. doi:10.1210/en.2015-1430

44. Wittmann G, Hrabovszky E, Lechan RM. Distinct glutamatergic and GABAergic subsets of hypothalamic pro-opiomelanocortin neurons revealed by in situ hybridization in male rats and mice. J Comp Neurol (2013) 521(14):3287-302. doi: 10.1002/cne.23350

45. Cheng CYY, Chu JYS, Chow BKC. Central and peripheral administration of secretin inhibits food intake in mice through the activation of the melanocortin system. Neuropsychopharmacology (2011) 36(2):459-71. doi:10.1038/ npp. 2010.178

46. Evans JM, Bey V, Burkey AR, Commons KG. Organization of endogenous opioids in the rostral agranular insular cortex of the rat. J Comp Neurol (2007) 500(3):530-41. doi:10.1002/cne.21197

47. Gotoh K, Liu M, Benoit SC, Clegg DJ, Davidson WS, D’Alessio D, et al. Apolipoprotein A-IV interacts synergistically with melanocortins to reduce food intake. Am J Physiol Regul Integr Comp Physiol (2006) 290(1):R202-7. doi:10.1152/ajpregu.00502.2005
48. Pandit R, Omrani A, Luijendijk MC, de Vrind VA, Van Rozen AJ, Ophuis RJ, et al. Melanocortin 3 receptor signaling in midbrain dopamine neurons increases the motivation for food reward. Neuropsychopharmacology (2016) 41(9):2241-51. doi:10.1038/npp.2016.19

49. Reyes BA, Glaser JD, Magtoto R, Van Bockstaele EJ. Pro-opiomelanocortin colocalizes with corticotropin-releasing factor in axon terminals of the noradrenergic nucleus locus coeruleus. Eur J Neurosci (2006) 23(8):2067-77. doi:10.1111/j.1460-9568.2006.04744.x

50. Singru PS, Wittmann G, Farkas E, Zseli G, Fekete C, Lechan RM. Refeedingactivated glutamatergic neurons in the hypothalamic paraventricular nucleus (PVN) mediate effects of melanocortin signaling in the nucleus tractus solitarius (NTS). Endocrinology (2012) 153(8):3804-14. doi:10.1210/en.2012-1235

51. Tavares E, Maldonado R, Minano FJ. Aminoprocalcitonin-mediated suppression of feeding involves the hypothalamic melanocortin system. Am J Physiol Endocrinol Metab (2013) 304(12):E1251-62. doi:10.1152/ajpendo.00590.2012

52. Zhan C, Zhou J, Feng Q, Zhang JE, Lin S, Bao J, et al. Acute and long-term suppression of feeding behavior by POMC neurons in the brainstem and hypothalamus, respectively. J Neurosci (2013) 33(8):3624-32. doi:10.1523/ JNEUROSCI.2742-12.2013

53. Desai M, Gayle D, Han G, Ross MG. Programmed hyperphagia due to reduced anorexigenic mechanisms in intrauterine growth-restricted offspring. Reprod Sci (2007) 14(4):329-37. doi:10.1177/1933719107303983

54. Mao S, Li X, Wang J, Ding X, Zhang C, Li L. miR-17-92 facilitates neuronal differentiation of transplanted neural stem/precursor cells under neuroinflammatory conditions. J Neuroinflammation (2016) 13(1):208. doi:10.1186/ s12974-016-0685-5

55. McGuckin CP, Jurga M, Miller AM, Sarnowska A, Wiedner M, Boyle NT, et al. Ischemic brain injury: a consortium analysis of key factors involved in mesenchymal stem cell-mediated inflammatory reduction. Arch Biochem Biophys (2013) 534(1-2):88-97. doi:10.1016/j.abb.2013.02.005

56. Banks WA, Coon AB, Robinson SM, Moinuddin A, Shultz JM, Nakaoke R, et al. Triglycerides induce leptin resistance at the blood-brain barrier. Diabetes (2004) 53(5):1253-60. doi:10.2337/diabetes.53.5.1253

57. Ahima RS, Hileman SM. Postnatal regulation of hypothalamic neuropeptide expression by leptin: implications for energy balance and body weight regulation. Regul Pept (2000) 92(1-3):1-7. doi:10.1016/S0167-0115(00)00142-7

58. Cottrell EC, Cripps RL, Duncan JS, Barrett P, Mercer JG, Herwig A, et al. Developmental changes in hypothalamic leptin receptor: relationship with the postnatal leptin surge and energy balance neuropeptides in the postnatal rat. Am J Physiol (2009) 296(3):R631-9. doi:10.1152/ajpregu.90690.2008

59. Delahaye F, Breton C, Risold PY, Enache M, Dutriez-Casteloot I, Laborie C, et al. Maternal perinatal undernutrition drastically reduces postnatal leptin surge and affects the development of arcuate nucleus proopiomelanocortin neurons in neonatal male rat pups. Endocrinology (2008) 149(2):470-5. doi:10.1210/en.2007-1263

60. Plagemann A, Harder T, Rake A, Voits M, Fink H, Rohde W, et al. Perinatal elevation of hypothalamic insulin, acquired malformation of hypothalamic galaninergic neurons, and syndrome $\mathrm{x}$-like alterations in adulthood of neonatally overfed rats. Brain Res (1999) 836(1-2):146-55. doi:10.1016/ S0006-8993(99)01662-5

61. Plagemann A, Harder T, Brunn M, Harder A, Roepke K, Wittrock-Staar M, et al. Hypothalamic proopiomelanocortin promoter methylation becomes altered by early overfeeding: an epigenetic model of obesity and the metabolic syndrome. J Physiol (2009) 587(Pt 20):4963-76. doi:10.1113/ jphysiol.2009.176156

62. Lee S, Lee KA, Choi GY, Desai M, Lee SH, Pang MG, et al. Feed restriction during pregnancy/lactation induces programmed changes in lipid, adiponectin and leptin levels with gender differences in rat offspring. J Matern Fetal Neonatal Med (2013) 26(9):908-14. doi:10.3109/14767058.2013.766686

63. Mela V, Diaz F, Vazquez MJ, Argente J, Tena-Sempere M, Viveros MP, et al. Interaction between neonatal maternal deprivation and serum leptin levels on metabolism, pubertal development, and sexual behavior in male and female rats. Biol Sex Differ (2016) 7:2. doi:10.1186/s13293-015-0054-6

64. Commins SP, Watson PM, Padgett MA, Dudley A, Argyropoulos G, Gettys TW. Induction of uncoupling protein expression in brown and white adipose tissue by leptin. Endocrinology (1999) 140(1):292-300. doi:10.1210/endo.140.1.6399

65. McCarthy MM, Arnold AP. Reframing sexual differentiation of the brain. Nat Neurosci (2011) 14(6):677-83. doi:10.1038/nn.2834 
66. Ikeda Y, Nagai A, Ikeda MA, Hayashi S. Sexually dimorphic and estrogendependent expression of estrogen receptor beta in the ventromedial hypothalamus during rat postnatal development. Endocrinology (2003) 144(11):5098-104. doi:10.1210/en.2003-0267

67. He Z, Ferguson SA, Cui L, Greenfield LJ, Paule MG. Development of the sexually dimorphic nucleus of the preoptic area and the influence of estrogen-like compounds. Neural Regen Res (2013) 8(29):2763-74. doi:10.3969/j. issn.1673-5374.2013.29.008

68. Frank MG, Hershman SA, Weber MD, Watkins LR, Maier SF. Chronic exposure to exogenous glucocorticoids primes microglia to pro-inflammatory stimuli and induces NLRP3 mRNA in the hippocampus. Psychoneuroendocrinology (2014) 40:191-200. doi:10.1016/j.psyneuen.2013.11.006
Conflict of Interest Statement: The authors declare that the research was conducted in the absence of any commercial or financial relationships that could be construed as a potential conflict of interest.

Copyright (C) 2017 Ziko, Sominsky, Nguyen, Yam, De Luca, Korosi and Spencer. This is an open-access article distributed under the terms of the Creative Commons Attribution License (CC BY). The use, distribution or reproduction in other forums is permitted, provided the original author(s) or licensor are credited and that the original publication in this journal is cited, in accordance with accepted academic practice. No use, distribution or reproduction is permitted which does not comply with these terms. 